\title{
La formación de investigadores en los departamentos de ciencias y tecnologías de la Universidad de Castilla-La Mancha
}

The researchers' education in the science-and-technology departments of the University of Castilla-La Mancha

\author{
Marisa PÉrez Aliende \\ Universidad Autónoma de Madrid \\ mp.aliende@uam.es
}

\begin{abstract}
Resumen
Se analiza la capacidad de la Universidad de CastillaLa Mancha para la formación de investigadores en las áreas de las ciencias y la tecnología entre 2002 y 2011. Para ello se ha estudiado la evolución en el número de estudiantes matriculados en programas de doctorado, de las tesis defendidas y dirigidas por profesores de los departamentos analizados, así como la productividad en tesis doctorales por profesor y departamento.
\end{abstract}

Palabras clave: Formación de investigadores. Tesis doctoral. Director de tesis. Universidades.

\section{Introducción}

Castilla-La Mancha durante sus primeros quince años de autonomía hizo un gran esfuerzo por mejorar su capacidad tecnológica, creciendo más que la media nacional; y, según Ramírez de Hijosa (1998), entre los factores que influyeron en su evolución destaca la Universidad. El entorno científico de Castilla-La Mancha está básicamente integrado por la Universidad de Castilla-La Mancha (UCLM). La universidad no se puede entender hoy en día sin la actividad investigadora; y en los últimos años hay que añadirle otra misión: la transferencia y explotación de los resultados de investigación (Sanz, 2003).

La investigación es el motor que hace avanzar el conocimiento en todos los campos del desarrollo, por lo que la formación de investigadores y de equipos de investigación es la herramienta imprescindible para que el avance se produzca (Gomar, 2002).

Si el Real Decreto 778/1998 que regulaba el tercer ciclo de estudios universitarios, la obtención y expedición del título de doctor y otros estudios de postgrado, recoge el papel de la universidad como responsable de la formación superior a todos los niveles para que la sociedad avance y, por lo tanto, con la responsabilidad y vocación de formar investigadores. Así mismo los estudios de tercer ciclo conducentes

\begin{abstract}
This paper reports the Castilla-La Mancha University capability to train researchers in science and technology in the period 2002-2012. The analyzed variables were the number of PhD students enrolled in doctorate programs, the number of doctoral thesis, defended and supervised by professors from the departments selected for this study, as well as the thesis productivity of supervisors and departments.
\end{abstract}

Keywords: Researchers' education. Doctoral thesis. Doctoral thesis supervisor. Universities.

a la obtención del título de doctor se realizarían bajo la supervisión y responsabilidad académica de un departamento, que además de proponer coordinaría los programas de doctorado. Posteriormente la ley orgánica 4/2007 expone cómo

[...] los estudios de doctorado se organizarán y realizarán en la forma que determinan los estatutos de las Universidades, de acuerdo con los criterios que para la obtención del título de doctor apruebe el Gobierno, previo informe del Consejo de Universidades.

Posteriormente, en el Real Decreto 99/2011 regulador de las enseñanzas oficiales de doctorado se considera al doctorado como un elemento fundamental en el punto de encuentro entre el Espacio Europeo de Educación Superior (EEES) y el Espacio Europeo de Investigación (EEI), "ambos pilares fundamentales de la sociedad basada en el conocimiento". La universidad sigue siendo la responsable de la formación doctoral en colaboración con otros entes implicados en la I+D+i. Encuadra a los doctorandos como investigadores en formación, considerando esta formación clave en una sociedad basada en el conocimiento, expresando textualmente: "El componente fundamental de la formación doctoral es el avance del conocimiento científico a través de la investigación original". 
La tesis doctoral es el documento que supone la culminación del ciclo de aprendizaje e investigación de un doctorando bajo la dirección y supervisión de uno o varios doctores (Fuentes y Arguimbau, 2010). El hecho de que esté sometida al análisis y valoración de un tribunal, compuesto por expertos en la materia, ofrece las garantías de cumplir con los requisitos necesarios exigidos por la investigación científica (López, 1996; Fernández y otros, 2003), y a su vez le acredita y abre las puertas al mundo de la investigación (Delgado y otros, 2006). Repiso, Torres y Delgado (2011) consideran como variable decisiva de la calidad y cantidad de tesis doctorales que se defienden, a la tradición científica que se materializa en estudios de postgrado y de doctorado. Mestre y Pérez Delgado (1991) afirman que

Las tesis doctorales constituyen una de las vías en las que se plasma parte de la investigación llevada a cabo en una Universidad, y por lo tanto es reflejo de las líneas de interés académico en diferentes momentos.

Pelechano (2002) aborda la dirección de tesis y su falta de reconocimiento ni como investigación, ni como docencia; sin embargo, se considera un indicativo de gran valor de la producción (Agudelo y otros, 2003) y no sólo un requisito para que se obtenga el título de doctor.

\section{La formación de investigadores en la UCLM}

Las tesis doctorales son el tipo de documento que mejor pueden caracterizar el potencial investigador de una institución, pues su futuro dependerá directamente de los doctorados y, a su vez, para éstos representa la culminación de todo un ciclo formativo (Torres, 2007). Por esta razón se van a estudiar en los siguientes apartados la evolución en el número de estudiantes de doctorado matriculados por curso académico y la finalización de su formación académica, materializada en forma de tesis doctoral, de la Universidad de Castilla-La Mancha en las áreas de ciencias y tecnologías. Para ello se analizan los 16 departamentos que abordan estas materias, desde el curso académico 2000-2001 hasta el 2011-2012.

En el presente trabajo para conocer la tendencia en la formación de investigadores por parte de la UCLM se han estudiado:

- La evolución en el número de estudiantes matriculados anualmente en programas de doctorado vinculados con los departamentos objeto de análisis.
- Evolución de las tesis doctorales defendidas anualmente en cada departamento.

- Dirección y codirección de tesis doctorales por departamentos.

- Productividad en tesis doctorales que se ha calculado dividiendo el número de tesis defendidas en un departamento entre la media del número de profesores funcionarios en el período de ese departamento. Para la obtención de este indicador de entre los profesores con dedicación a tiempo completo se han considerado únicamente a los profesores funcionarios con categoría de catedráticos y titulares de universidad, así como catedráticos de escuela universitaria; no se han contabilizado a los titulares de escuela universitaria porque no necesitan el título de doctor para conseguir la plaza, con lo que no tienen capacidad investigadora, a excepción de los que sí son doctores (Buela Casal y Castro, 2008).

Los datos empleados proceden de fuentes institucionales, por un lado las memorias de investigación que la UCLM edita con periodicidad anual, y por otro la información proporcionada por el Servicio de Alumnos, en lo relativo a la matrícula en los diferentes programas de doctorado.

\subsection{Evolución del número de estudiantes matriculados en programas de doctorado}

La evolución de la matrícula en relación con el total de matriculados en programas de doctorado de la UCLM y los vinculados con los departamentos objeto de estudio en el presente trabajo se puede ver en la tabla I. En el curso 20002001 los segundos representaban únicamente el $15,64 \%$ de todos los doctorandos mientras que en $2011-2012$ son el $44,39 \%$, llegando a representar en el curso $2009-2010$ el $49 \%$ del total de estudiantes matriculados en programas de doctorado de la UCLM.

En relación con el número de estudiantes que anualmente se matriculan en cursos de doctorado vinculados a programas impartidos por alguno de los departamentos analizados en este artículo, podemos decir que la tendencia es ascendente, experimentando un incremento constante desde el curso 2000-2001 —en el que se matricularon 96 personas- hasta el 20082009 -en el que se alcanza el máximo número, 518, descendiendo posteriormente y estabilizándose en los dos últimos cursos analizados en torno a 485 estudiantes (figura 1)-. 


\begin{tabular}{|c|c|c|c|}
\hline \multirow[t]{3}{*}{ Año } & \multicolumn{2}{|c|}{ Matrícula doctorandos } & \multirow[t]{3}{*}{$\%$} \\
\hline & Vinculado a & UCLM & \\
\hline & dptos. analizados & & \\
\hline Curso 2000-2001 & 96 & 614 & 15,64 \\
\hline Curso 2001-2002 & 132 & 592 & 22,30 \\
\hline Curso 2002-2003 & 166 & 657 & 25,27 \\
\hline Curso 2003-2004 & 236 & 764 & 30,89 \\
\hline Curso 2004-2005 & 237 & 754 & 31,43 \\
\hline Curso 2005-2006 & 338 & 900 & 37,56 \\
\hline Curso 2006-2007 & 340 & 933 & 36,44 \\
\hline Curso 2007-2008 & 348 & 979 & 35,55 \\
\hline Curso 2008-2009 & 518 & 1329 & 38,98 \\
\hline Curso 2009-2010 & 468 & 954 & 49,06 \\
\hline Curso 2010-2011 & 485 & 1162 & 41,74 \\
\hline Curso 2011-2012 & 487 & 1097 & 44,39 \\
\hline
\end{tabular}

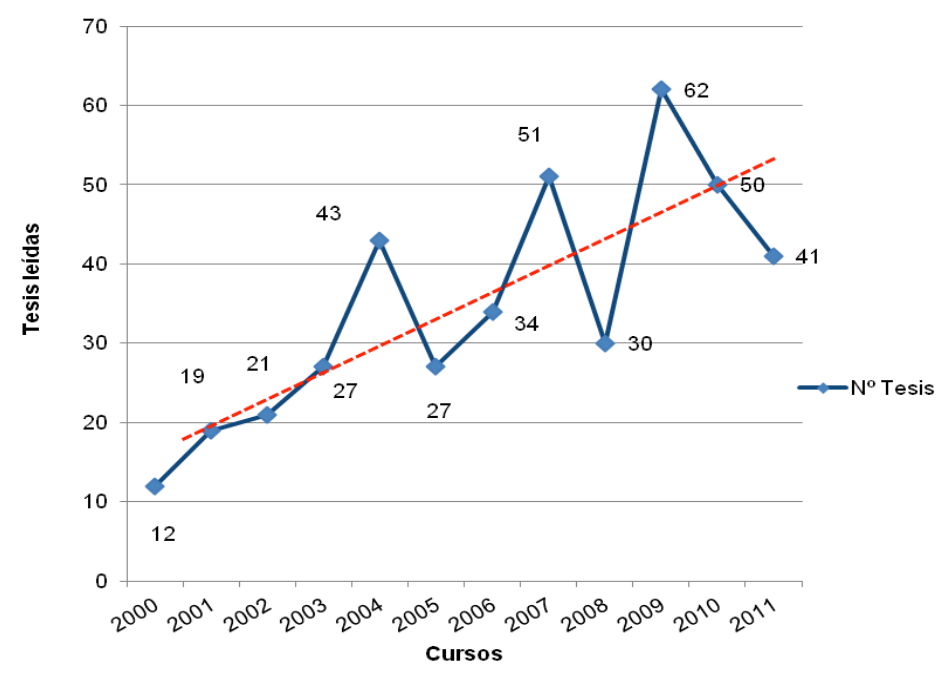

Tabla I. Evolución del número de estudiantes matriculados en programas de doctorado vinculados con los departamentos analizados y de la UCLM

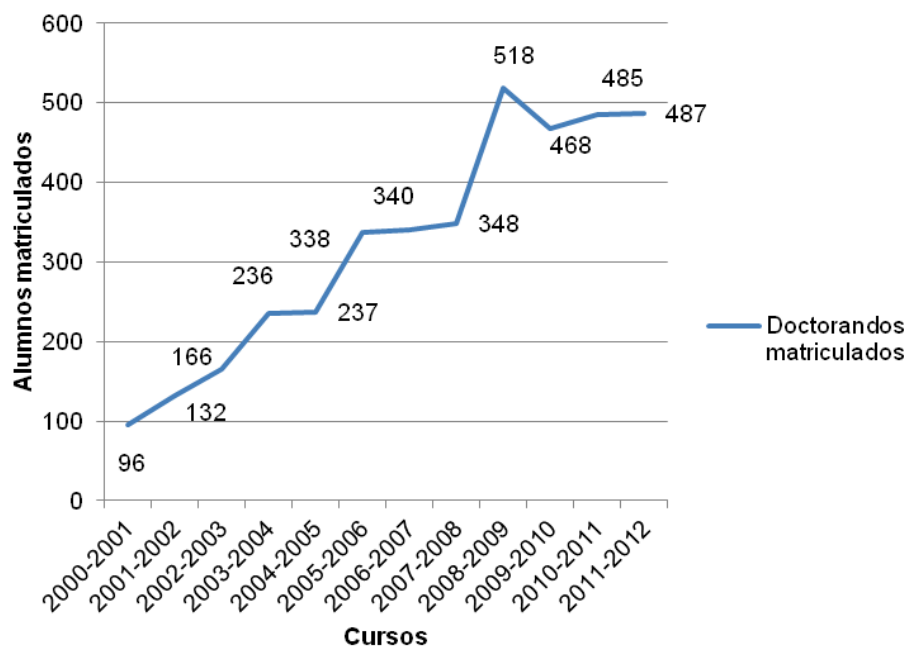

Figura 1. Evolución en la matrícula en los doctorados impartidos por los departamentos objeto de estudio

2.2. Evolución de las tesis defendidas en la UCLM por departamento

Durante todo el período, en los 16 departamentos de la UCLM se han defendido un total de 417 tesis. La tendencia general en la defensa de tesis es ascendente (figura 2), si bien se van produciendo altibajos: en el año 2000 se defendieron 12 tesis doctorales y en 2011, 41. El año 2000 fue en el que se leyeron menos tesis (12), mientras que en el que más se leyeron fue 2009 (62), descendiendo progresivamente en los dos años siguientes hasta alcanzar valores próximos a 2004.

Figura 2. Número de tesis leídas en los departamentos de la UCLM analizados

\begin{tabular}{ll}
\hline Departamento & $N^{\circ}$ \\
\hline TSI & 63 \\
\hline QIOB & 53 \\
\hline SIS & 44 \\
\hline CTA & 43 \\
\hline MAIP & 41 \\
\hline IEEAC & 39 \\
\hline CM & 37 \\
\hline QATA & 36 \\
\hline QF & 35 \\
\hline IQ & 33 \\
\hline PVTA & 29 \\
\hline MAT & 26 \\
\hline CA & 23 \\
\hline FA & 10 \\
\hline ICE & 9 \\
\hline IGM & 9 \\
\hline Total & 530 \\
\hline
\end{tabular}

Tabla II. Direcciones y codirecciones de tesis por departamento durante el período 2000-2011

(Acrónimos en apéndice)

La tabla II recoge el total de tesis dirigidas y codirigidas por los profesores de los departamentos analizados durante el período 20002011, que ha supuesto un total de 530 tesis doctorales. Destacan Tecnologías y Sistemas de Información (TSI) por dirigir un total de 63, seguido de Química Inorgánica, Orgánica y Bioquímica (QIOB) con 53 y Sistemas Informáticos (SIS) con 44 tesis. 
Entre los departamentos que han dirigido un número inferior a diez tesis en todo el período se encuentran Ingeniería Geológica y Minera (IGM), Ingeniería Civil y de la Edificación (ICE) con 9 respectivamente, y Física Aplicada (FA) con 10.

En la tabla III se analiza el número de tesis defendidas anualmente en cada uno de los departamentos. Como se ha indicado anteriormente, se han defendido un total de 417 tesis en el conjunto de los departamentos. En trece de los departamentos se han defendido como mínimo 10 tesis, siendo el más productivo Química Inorgánica, Orgánica y Bioquímica (QIOB) con 51 , y el que menos Ingeniería Geológica y Minera (IGM) con seis tesis defendidas. Tecnologías y Sistemas de Información (TSI) es el segundo departamento en el que más tesis se defienden (49), contabilizándose hasta 15 en un mismo año (2009).

\begin{tabular}{lccccccccccccc}
\hline Dept. & 00 & 01 & 02 & 03 & 04 & 05 & 06 & 07 & 08 & '09 & '10 & '11 & Total \\
\hline QIOB & 5 & 3 & 3 & 4 & 3 & 5 & 5 & 8 & 2 & 8 & 3 & 2 & 51 \\
\hline TSI & 1 & 3 & 4 & 1 & 0 & 1 & 3 & 9 & 1 & 15 & 8 & 3 & 49 \\
\hline CTA & 1 & 2 & 1 & 3 & 7 & 3 & 3 & 3 & 4 & 9 & 3 & 2 & 41 \\
\hline SIS & 0 & 2 & 1 & 3 & 2 & 3 & 4 & 8 & 1 & 5 & 7 & 4 & 40 \\
\hline IQ & 2 & 1 & 1 & 4 & 4 & 2 & 2 & 6 & 3 & 2 & 4 & 2 & 33 \\
\hline MAIP & 0 & 0 & 1 & 2 & 3 & 2 & 6 & 5 & 4 & 3 & 4 & 2 & 32 \\
\hline QF & 0 & 2 & 3 & 2 & 4 & 0 & 2 & 3 & 0 & 5 & 4 & 5 & 30 \\
\hline QATA & 0 & 5 & 4 & 4 & 4 & 5 & 1 & 0 & 0 & 2 & 2 & 2 & 29 \\
\hline PVTA & 2 & 0 & 1 & 2 & 6 & 1 & 0 & 2 & 4 & 3 & 2 & 3 & 26 \\
\hline IEEAC & 1 & 1 & 0 & 0 & 2 & 1 & 4 & 1 & 5 & 1 & 4 & 3 & 23 \\
\hline CM & 0 & 0 & 0 & 0 & 1 & 1 & 0 & 2 & 3 & 2 & 3 & 6 & 18 \\
\hline MAT & 0 & 0 & 0 & 1 & 1 & 1 & 2 & 2 & 2 & 3 & 1 & 1 & 14 \\
\hline CA & 0 & 0 & 0 & 0 & 3 & 1 & 1 & 1 & 0 & 3 & 0 & 2 & 11 \\
\hline FA & 0 & 0 & 2 & 0 & 2 & 0 & 0 & 1 & 0 & 0 & 1 & 1 & 7 \\
\hline ICE & 0 & 0 & 0 & 1 & 0 & 1 & 1 & 0 & 0 & 1 & 1 & 2 & 7 \\
\hline IGM & 0 & 0 & 0 & 0 & 1 & 0 & 0 & 0 & 1 & 0 & 3 & 1 & 6 \\
\hline Total & 12 & 19 & 21 & 27 & 43 & 27 & 34 & 51 & 30 & 62 & 50 & 41 & 417 \\
\hline
\end{tabular}

Tabla III. Evolución de las tesis defendidas en los diferentes departamentos

\subsection{Productividad en tesis doctorales por profesor y departamento}

En este apartado se estudia la productividad de cada departamento en tesis doctorales. Entre los departamentos con mayor productividad de tesis se encuentran los dos del área de la Informática: En primer lugar, Tecnologías y Sistemas de Información (TSI) que con 49 tesis defendidas y una media de 15 profesores, presenta una proporción de 3,34 tesis por profesor. El segundo sería Ciencia y Tecnología Agroforestal y Genética (CTA) con 41 tesis y 17 profesores alcanzan una productividad de 2,35 y en tercer lugar Sistemas Informáticos (SIS) que cuenta con 40 tesis y 18 profesores teniendo una productividad del 2,18 tesis por profesor. En sexta posición y con el mayor número de tesis dirigidas (51) se encuentra el departamento de Química Inorgánica, Orgánica y Bioquímica (QIOB), sin embargo el promedio de profesores también es el más elevado, 35 por lo que cuenta con una productividad más baja, 1,45 tesis por profesor.

Los dos departamentos con una menor productividad son: Física Aplicada (FA) con 0,51 tesis defendidas por profesor, e Ingeniería Geológica y Minera (IGM) con 0,88 (tabla IV, en la página siguiente). El departamento con una mayor productividad en uno de los años, concretamente 2002, fue el de Tecnologías y Sistemas de Información (TSI), con una tesis por profesor. La productividad en tesis doctorales de los departamentos de ciencias y tecnologías de la UCLM es de 1,51 tesis por profesor.

\section{Discusión}

En términos cuantitativos la formación de nuevos investigadores en España resulta claramente inferior a la media europea (Fuentes y Arguimbau, 2010). Iribarren (2006) considera que la 
vinculación de un departamento con un programa de doctorado o máster puede incidir en su productividad, pues se invierte gran cantidad de tiempo en la dirección y supervisión de tesis y tesinas; por el contrario, el no contar con este nivel de docencia puede acarrear problemas a la hora de encontrar titulados que muestren interés por sus líneas de investigación. La formación de doctores (investigadores) es una de las tareas más complicadas de la universidad. De Miguel y otros investigadores (2004) consideran que nos encontramos ante un sistema endogámico con programas de doctorado que no son programas de formación de personal investigador, sino más bien de formación de personal docente propio, con estudiantes que se convierten en profesores de la misma universi- dad antes de terminar el doctorado. Con doctores cuya tesis doctoral es en muchas ocasiones la última investigación que realizan en una carrera docente y con una tasa de abandono de los programas de doctorado muy elevada. De manera diferente, Carabaña (2004) considera que la razón principal por la cual en un país como España hay poca investigación no es por la falta de inversión, sino por la falta de una élite académica de alto nivel de la cual extraer investigadores competitivos que consigan dinero. Si bien, en ambos trabajos se considera que la tesis doctoral en muchos momentos pasa de ser un ejercicio de investigación a una formalidad para progresar en la carrera docente.

\begin{tabular}{|c|c|c|c|c|c|c|c|c|c|c|c|c|c|c|c|}
\hline \multirow[t]{2}{*}{ Dept. } & \multicolumn{12}{|c|}{ Tesis leídas / Profesores funcionarios } & \multirow{2}{*}{$\begin{array}{l}\text { Total } \\
\text { tesis } \\
(\mathrm{T})\end{array}$} & \multirow{2}{*}{$\begin{array}{l}\text { Media } \\
\text { prof. } \\
(\mathrm{P})\end{array}$} & \multirow{2}{*}{$\begin{array}{c}\text { Produc- } \\
\text { tividad } \\
(\mathrm{T} / \mathrm{P})\end{array}$} \\
\hline & 2000 & 2001 & 2002 & 2003 & 2004 & 2005 & 2006 & 2007 & 2008 & 2009 & 2010 & 2011 & & & \\
\hline TSI & 0,25 & 0,75 & 1,00 & 0,08 & 0,00 & 0,08 & 0,23 & 0,64 & 0,04 & 0,63 & 0,32 & 0,11 & 49 & 14,67 & 3,34 \\
\hline CTA & 0,07 & 0,13 & 0,06 & 0,17 & 0,30 & 0,19 & 0,23 & 0,21 & 0,22 & 0,45 & 0,15 & 0,10 & 41 & 17,42 & 2,35 \\
\hline SIS & 0,00 & 0,25 & 0,11 & 0,18 & 0,12 & 0,19 & 0,25 & 0,47 & 0,04 & 0,19 & 0,23 & 0,12 & 40 & 18,33 & 2,18 \\
\hline IQ & 0,14 & 0,07 & 0,07 & 0,22 & 0,22 & 0,12 & 0,12 & 0,32 & 0,14 & 0,08 & 0,14 & 0,07 & 33 & 19,58 & 1,69 \\
\hline MAIP & 0,00 & 0,00 & 0,06 & 0,10 & 0,15 & 0,10 & 0,30 & 0,25 & 0,18 & 0,13 & 0,15 & 0,07 & 32 & 20,17 & 1,59 \\
\hline QIOB & 0,19 & 0,11 & 0,10 & 0,12 & 0,09 & 0,15 & 0,14 & 0,23 & 0,05 & 0,19 & 0,07 & 0,04 & 51 & 35,17 & 1,45 \\
\hline PVTA & 0,17 & 0,00 & 0,07 & 0,13 & 0,38 & 0,06 & 0,00 & 0,11 & 0,18 & 0,13 & 0,08 & 0,11 & 26 & 18,67 & 1,39 \\
\hline IEEAC & 0,17 & 0,14 & 0,00 & 0,00 & 0,14 & 0,07 & 0,27 & 0,06 & 0,24 & 0,04 & 0,15 & 0,11 & 23 & 16,67 & 1,38 \\
\hline QF & 0,00 & 0,12 & 0,17 & 0,10 & 0,19 & 0,00 & 0,10 & 0,14 & 0,00 & 0,18 & 0,14 & 0,17 & 30 & 22,00 & 1,36 \\
\hline QATA & 0,00 & 0,29 & 0,24 & 0,18 & 0,18 & 0,23 & 0,05 & 0,00 & 0,00 & 0,07 & 0,07 & 0,07 & 29 & 22,25 & 1,30 \\
\hline ICE & 0,00 & 0,00 & 0,00 & 0,20 & 0,00 & 0,25 & 0,25 & 0,00 & 0,00 & 0,13 & 0,11 & 0,20 & 7 & 6,08 & 1,15 \\
\hline $\mathrm{CM}$ & 0,00 & 0,00 & 0,00 & 0,00 & 0,06 & 0,05 & 0,00 & 0,11 & 0,17 & 0,09 & 0,12 & 0,24 & 18 & 17,00 & 1,06 \\
\hline MAT & 0,00 & 0,00 & 0,00 & 0,08 & 0,08 & 0,06 & 0,13 & 0,13 & 0,13 & 0,16 & 0,05 & 0,04 & 14 & 15,25 & 0,92 \\
\hline $\mathrm{CA}$ & 0,00 & 0,00 & 0,00 & 0,00 & 0,20 & 0,07 & 0,07 & 0,07 & 0,00 & 0,20 & 0,00 & 0,13 & 11 & 12,08 & 0,91 \\
\hline IGM & 0,00 & 0,00 & 0,00 & 0,00 & 0,14 & 0,00 & 0,00 & 0,00 & 0,13 & 0,00 & 0,38 & 0,13 & 6 & 6,83 & 0,88 \\
\hline FA & 0,00 & 0,00 & 0,17 & 0,00 & 0,14 & 0,00 & 0,00 & 0,08 & 0,00 & 0,00 & 0,05 & 0,05 & 7 & 13,83 & 0,51 \\
\hline Total & & & & & & & & & & & & & 417 & 276 & 1,51 \\
\hline
\end{tabular}

Tabla IV. Productividad en tesis doctorales por profesor y departamento (anual y media del período)

El estudio de las tesis doctorales leídas en una universidad es uno de los aspectos a tener en cuenta al realizar un análisis de las capacidades científico-técnicas de los centros españoles de I+D (Sanz-Menéndez, Meza y Barrios, 2002). Las tesis proporcionan una imagen fiel de las nuevas vías de investigación que van surgiendo (Fuentes y Arguimbau, 2010; Delgado y otros, 2006) y resultan útiles para calibrar el potencial de formación de nuevos investigadores de un sistema, así como la productividad científica de sus directores (Buela-Casal, 2005). De esta manera se puede determinar quienes han sido los directores que han dirigido a un mayor número de doctorandos en un período determinado y así saber si son directores ocasionales o muy productivos (De Filippo, 2008). La elección del director de tesis resulta un factor clave tanto para el éxito de la empresa como para la formación de futuras escuelas científicas (Delgado y otros, 2006).

Pérez Aliende (2012), en el estudio realizado sobre la UCLM, concluía que muestra un perfil científico donde destaca la producción en tres 
áreas científicas: Informática, Ingeniería y Química. El departamento de Química Inorgánica, Orgánica y Bioquímica junto con los dos departamentos del área de Informática eran los que contaban con una mayor producción científica en el período analizado. Departamentos que son los que cuentan con un mayor número de direcciones y codirecciones de tesis. Atendiendo al hecho de que la Universidad se encuentra asentada en una comunidad eminentemente agrícola y al interés que suscita todo lo relacionado con la Agroalimentación (Chinchilla y Olmeda, 2010), hay que destacar la actividad del departamento de Ciencia y Tecnología Agroforestal y Genética (CTA), que se encuentra entre los que más tesis dirigen y es el segundo departamento en productividad de tesis doctorales.

No en vano la productividad en tesis doctorales por profesor es un criterio utilizado tanto en rankings internacionales como en nacionales. Buela-Casal junto con otros autores elaboran anualmente el "ranking en investigación de las universidades públicas españolas" incluyendo el criterio de tesis doctorales y utilizando como indicador la productividad, entendida como la proporción de tesis doctorales por profesores funcionarios de cada una de las universidades públicas en un período de cinco cursos.

La UCLM ha ido escalando posiciones progresivamente: Si en el ranking 2008 de universidades públicas españolas, según el criterio de tesis doctorales, obtuvo la posición 29 de 48 universidades, considerando las tesis doctorales defendidas desde el curso académico 2002/2003 hasta el 2006/2007; en el ranking de 2009 alcanzó la posición 26, para las tesis doctorales defendidas entre el curso académico $2003 / 2004$ y el $2007 / 2008$; en el 2010 , la 23 , para las tesis defendidas en el quinquenio 2004/2005-2008/2009; y la 16 en el ranking 2011.

En las cuatro ediciones del ranking, la Universidad Autónoma de Barcelona se ha situado entre las tres primeras, y destaca como la Universidad Pompeu Fabra ha ido escalando posiciones hasta convertirse en la primera en 2010 y 2011 (Buela-Casal y otros, 2009, 2010, 2011 y 2012). Quevedo Blasco y otros autores (2010) realizaron un estudio con el objetivo de evaluar el rendimiento de los doctorados en universidades públicas a través de las tesis doctorales, concluyendo que existe una correlación entre poseer un bajo número de doctorados con mención de calidad y tener una reducida producción de tesis doctorales, como era el caso de las universidades de Jaén, Castilla-La Mancha y Burgos, entre otras.

\section{Conclusiones}

El porcentaje de estudiantes matriculados en programas de doctorado en el conjunto de la Universidad ha experimentado un incremento desde el curso 2000-2001 hasta el 2011-2012 del $78,66 \%$, mientras que el de los matriculados en programas vinculados a los departamentos del presente estudio ha sido del $407,29 \%$. Por lo que se puede decir que en la última década se ha "disparado" el número de doctorandos en las áreas de ciencias y tecnologías de la Universidad.

Los departamentos con mayor número de profesores que dirigen o codirigen tesis doctorales pertenecen al ámbito de la Informática y de la Química: Tecnologías y Sistemas de Información, seguido de Química Inorgánica Orgánica y Bioquímica, y Sistemas Informáticos. Sin olvidar el sector de la agroalimentación representado por Ciencia y Tecnología Agroforestal y Genética..

El departamento de Química Inorgánica Orgánica y Bioquímica es en el que más tesis se defienden, aunque se ha observado que entre los tres departamentos con mayor productividad de tesis doctorales se encuentran los dos del área de Informática, Tecnologías y Sistemas de Información y Sistemas Informáticos.

Para comprobar si se ha producido o no endogamia sería necesario realizar un estudio más pormenorizado que nos permitiera comparar, entre otros aspectos, la correlación existente entre obtener el título de doctor en la Universidad y posteriormente obtener una plaza de titular.

\section{Referencias}

Agudelo, D.; Bretón López, J.; Ortiz Recio, G.; Poveda Vera, J.; Teva, I.; Valor Segura, I.; Vico, C. (2003). Análisis de la productividad científica de la Psicología española a través de las tesis doctorales. // Psicothema. 15 (2003) 595-609.

Buela Casal, G. (2005). Situación actual de la productividad científica de las universidades españolas. // International Journal of Clinical and Health Psychology. 5:1 (2005) 175-190.

Buela Casal, G.; Bermúdez, M. P.; Sierra, J. C.; QuevedoBlasco, R.; Castro, Á. (2009). Ranking de 2008 en productividad en investigación de las universidades públicas españolas. // Psicothema. 21:2 (2009) 309-317.

Buela Casal, G.; Bermúdez, M. P.; Sierra, J. C.; QuevedoBlasco, R.; Castro, Á. (2010). Ranking de 2009 en investigación de las universidades públicas españolas. // Psicothema. 22:2 (2010) 171-179.

Buela Casal, G.; Bermúdez, M. P.; Sierra, J. C.; QuevedoBlasco, R.; Castro, Á.; Guillén-Riquelme, A. (2011). Ranking de 2010 en producción y productividad en investigación de las universidades públicas españolas. // Psicothema. 23:4 (2011) 527-536. 
Buela Casal, G.; Bermúdez, M. P.; Sierra, J. C.; QuevedoBlasco, R.; Castro, Á.; Guillén-Riquelme, A. (2012). Ranking de 2011 en producción y productividad en investigación de las universidades públicas españolas. // Psicothema. 24:4 (2012) 505-515.

Buela Casal, G.; Castro, Á. (2008). Análisis de la evolución de los programas de doctorado con mención de calidad en las universidades españolas y pautas para su mejora. // Revista de Investigación en Educación. 5 (2008) 49-60.

Carabaña, J. (2004). Industria de investigación y producción de doctores. // Empiria: revista de metodología de ciencias sociales. 8 (2004) 163-180.

Chinchilla Rodríguez, Z.; Olmeda Gómez, C. (2010). Producción y colaboración científica en agroalimentación. // Sanz Menéndez, L.; Cruz Castro, L. (comp.). Análisis sobre Ciencia e Innovación en España. Madrid: Ministerio de Ciencia e Innovación y Fecyt, 2010. 366-399. http://www.investigacion.cchs.csic.es/dci/sites/investigac ion.cchs.csic.es.dci/files/Analisis Ciencia_Innovacion. pdf (5/12/2011).

De Filippo, D. (2008). Movilidad y producción científica en la UC3M: Estudio de la actividad científica del profesorado a partir de bases de datos institucionales (Universitas XXI) y bibliográficas (WoS, ISOC, ICYT) (1997-2005). Getafe: Universidad Carlos III, 2008. Tesis doctoral.

De Miguel, J. M.; Sarabia, B.; Vaquera, E. G.; Amirah, H. (2004). ¿Sobran o faltan doctores?. // Empiria: Revista de metodología en ciencias sociales. 7 (2004) 115-155.

Delgado López-Cózar, E.; Torres Salinas, D.; Jiménez Contreras, E.; Ruíz Pérez, R. (2006). Análisis bibliométrico y de redes sociales aplicado a las tesis bibliométricas defendidas en España (1976-2002): temas, escuelas científicas y redes académicas. // Revista Española de Documentación Científica. 29:4 (2006).

Fernández Cano, A.; Torralbo, M.; Rico, L.; Gutiérrez, P.; Maz, A. (2003). Análisis cienciométrico de las tesis doctorales españolas en educación matemática (19761998). // Revista Española de Documentación Científica. 26:2 (2003) 162-176.

Fuentes Pujol, E.; Arguimbau Vivó, L. (2010). Las tesis doctorales en España (1997-2008): análisis, estadísticas y repositorios cooperativos. // Revista Española de Documentación Científica. 33:1 (2010) 63-89.

Gomar Sancho, C. (2002). La tesis doctoral, ¿a quién sirve y para qué se utiliza?. // Rev. Esp. Anestesiol. Reanim. 49:3 (2002) 121-123.

Iribarren Maestro, I. (2006). Producción científica y visibilidad de los investigadores de la Universidad Carlos III de Madrid en las bases de datos del ISI 1997-2003. Getafe: Universidad Carlos III, 2006. Tesis doctoral.

López López, P. (1996). Introducción a la bibliometría. Valencia: Promolibro, 1996

Mestre, V.; Pérez Delgado, E. (1991). La psicología española a través de las tesis doctorales sobre psicología en las universidades españolas. // Revista de Historia de la Psicología. 12:2 (1991) 59-72.

Pelechano, V. (2002). Valoración de la actividad científica en psicología, ¿pseudoproblema, sociologismo o idealismo?. // Análisis y modificación de conducta. 28:119 (2002) 323-362.

Pérez Aliende, M. L. (2012). Evaluación de la actividad científica de los departamentos de ciencias y tecnologías de la Universidad de Castilla-La Mancha, a partir de la Web of Science (2000-2007). Getafe: Universidad Carlos III, 2012. Tesis doctoral.

Quevedo Blasco, R.; Río Bermúdez, L. del, Bermúdez Sánchez, M. P.; Buela Casal, G. (2010). Relación entre los programas de doctorado con mención de calidad y la productividad de tesis doctorales. // Revista Digital Universitaria. 11:5 (2010).

Ramírez Hijosa, J. J. (1998). La emergencia de un Sistema Regional de Innovación. // Añil: Cuadernos de CastillaLa Mancha. 15 (1998) 19-20.

Repiso, R.; Torres, D.; Delgado, E. (2011). Análisis bibliométrico y de redes sociales en tesis doctorales españolas sobre televisión (1976-2007). // Comunicar: revista científica de comunicación y educación. (2011). http://dx.doi.org/10.3916/C37-2011-03-07 (1/8/2011).

Sanz Menéndez, L. (2003). La investigación en la universidad española: la financiación competitiva de la investigación, con especial referencia a las ciencias sociales y económicas. http://hdl.handle.net/10261/1568 ( 1/9/2011).

Sanz Menéndez, L.; Meza, R.; Barrios, P. (2002). Identificación de los centros de $\mathrm{i}+\mathrm{d}$ con mayores capacidades científico-técnicas en las diversas comunidades autónomas: a partir de la obtención de ayudas para la ejecución de proyectos de $\mathrm{i}+\mathrm{d}$ otorgadas por la dirección general de investigación mediante convocatoria pública por concurrencia competitiva. Análisis de las convocatorias desde 1996 hasta 2001. Madrid: Ministerio de Ciencia y Tecnología. Dirección General de Investigación, 2002.

Torres Salinas, D. (2007). Diseño de un sistema de información y evaluación científica. Análisis cienciométrico de la actividad investigadora de la Universidad de Navarra en el área de ciencias de la salud. 1999-2005. Granada: Universidad de Granada, 2007. Tesis doctoral.

\section{Apéndice}

\begin{tabular}{ll}
\hline Abrev. & Departamento \\
\hline CA & Ciencias Ambientales \\
\hline CM & Ciencias Médicas \\
\hline CTA & Ciencia y Tecnología Agroforestal y Genética \\
\hline FA & Física Aplicada \\
\hline ICE & Ingeniería Civil y de la Edificación \\
\hline IEEA & Ingeniería Eléctrica, Electrónica, Automática y \\
C & Comunicaciones \\
\hline IGM & Ingeniería Geológica y Minera \\
\hline INFO & Informática \\
R & \\
\hline IQ & Ingeniería Química \\
\hline MAIP & Mecánica Aplicada e Ingeniería de Proyectos \\
\hline MAT & Matemáticas \\
\hline PVT & Producción Vegetal y Tecnología Agraria \\
A & \\
\hline QAT & Química Analítica y Tecnología de los Alimentos \\
A & \\
\hline QF & Química Física \\
\hline QIOB & Química Inorgánica, Orgánica y Bioquímica \\
\hline SIS & Sistemas Informáticos \\
\hline TSI & Tecnologías y Sistemas de Información \\
\hline
\end{tabular}

Tabla V. Abreviaturas de los departamentos de la UCLM

Enviado: 2012-04-05. Segunda versión: 2013-07-14. Aceptado: 2013-08-24. 
